\title{
A Cascade Algorithm Combined Kohonen Feature Map with Fuzzy C-Means Applied in MR Brain Image Segmentation
}

\author{
Chung-Chih Lin, *Jeng-Ren Duann, **Hui-Cheng Cheng, Jyh-Horng Chen \\ The Institute of Electrical Engineering, National Taiwan University \\ *The Institure of Applied Physics, Chung Yuan Christian University \\ **Department of Radiology, Taipei Veterans General Hospital
}

Abstract-In this study, a cascade algorithm combined Kohonen feature map with FCM was applied to segment the MR brain images. The method we proposed was proved has better performance than FCM method usually used in image segmentation researches. Because the algorithm is unsupervised, it can reduce the errors caused by intraobserver and interobserver estimation. In this paper, we also utilized the MR images acquired by PAIR protocol to verify the result of the image segmentation.

\section{Introduction}

THE FCM(fuzzy c-mans, FCM) can be utilized to solve the clustering, feature selection, medical diagnosis, and automatic target recognition [1], but it always needs a large number of memory to keep the data set and also requires a large amount of CPU time to compute. In order to conquer these drawbacks, we propose a cascade algorithm combined Kohonen feature map[2] with FCM to accelerate the computation and reduce the memory required to perform the image segmentation process.

\section{Methods and Materials}

An unsupervised algorithm Kohonen feature map of twolayer architecture was used to classify the pixels in the MR brain images into categories of gray matter, white matter, and cerebrospinal fluid (CSF). The processes learning algorithm of Kohonen feature map is

$$
\Delta W_{i j}=\eta \times\left(X_{i}-W_{i j}\right) \times e^{-\frac{D i s t a n c e}{R}}
$$

where $\eta$ is the learning rate of the network, $\mathrm{R}$ is the radium of convergence, and Distance is the distance between the winner and other output nodes. When all of the output nodes were updated according to the learning algorithm, the average distortion was calculated to be as the criteria of the convergence of the system. The equation to calculate the average distortion is

$$
\begin{aligned}
& D_{m}=\sum_{i}^{P i x e l} \sum_{j}^{\text {Vector }}\left|X_{i, j}-W_{\text {winner }, j}\right| \\
& \frac{\left|D_{m}-D_{m+1}\right|}{D_{m}}<\varepsilon
\end{aligned}
$$

where $x_{i j}$ is the $j$ th vector of the ith input unit, $w_{\text {winner } j}$ is the weighting vector between the winner node in the output layer and all nodes in the input layer, and $\varepsilon$ is the criteria of convergence. In this study, the parameter $\varepsilon$ was set as $10^{-4}$. After the preprocess of the Kohonen feature map, the result of the output layer will be set to the parameters of the FCM to label the tissues in the image. The structure of the experiment is shown in Fig. 1. All algorithms were implemented in $C$ language and run on Pentium-133 personal computer.

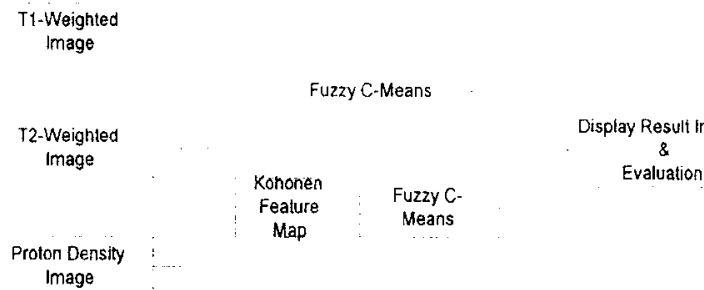

Fig. 1 The structure of this experiment

The MR brain images of a $28 \mathrm{yr}$. healthy male acquired by T1 weighted, $T 2$ weighted and PD weighted(Fig. 2), respectively, were used to train the Kohonen feature map and conventional FCM for image segmentation.

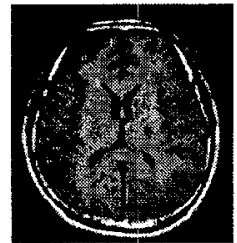

T1-Weighted

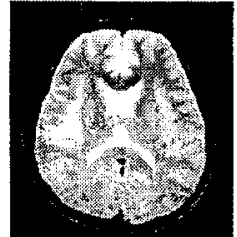

T2-Weighted

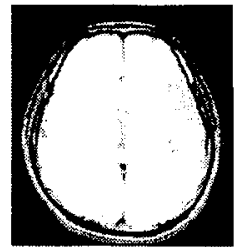

Proton Density
Fig. 2 Original images

Finally, Image segmented by the Kohonen feature map were examined by clinician and in comparison with a reference boundary image acquired by the PAIR (PArtial volume senstised Inversion Recovery, PAIR)[3] pulse sequence. The PAIR protocol was achieved by choosing TI properly to set the transition boundary between two different tissues of interest as nulling points to outline the tissues with a black profile.

\section{Results}

The time needed to train the conventional FCM image segmentation method exceeds 44 minutes. The computation 
times of the method proposed in this paper would vary with the size of the output layer of Kohonen feature map. They were 13 mins., 20 mins., 25 mins., and 37 mins., respectively, when the output mesh is from 6 by 6 to 9 by 9 . The result indicates that Kohonen feature map with 8 by 8 output layer has the best quality/time ratio than others (Fig. 3).

Because the gray matter and the boundary between white matter and CSF will be set to null by the PAIR protocol $(\mathrm{TI}=2500 \mathrm{~ms}$ ), the image could be used as the reference to verify the result of image segmentation. The result showed in Fig. 4 indicates that the images segmented by Kohonen feature map and FCM can match the PAIR inage. However, Kohonen feature map is better than FCM in noise immunity, because there is few spike in the image segmented by feature map method.

In order to verify the reliability of the result of the proposed method in this study, different image slices obtained from the same volunteer and obtained from the different volunteers (26 yr. healthy male) were tested and the comparison was made. In Fig. 5 and Fig. 6, gray matter, white matter, and CSF can be classified clearly.

\section{Discussions}

Increasing the number of the output layer nodes of the Kohonen feature map makes the result of classification more accurate. But the performance and accuracy of the image segmentation by Kohonen feature map method is not proportional to the number of output layer nodes. The result shows that when the mesh of the output layer is 8 by 8 , the Kohonen feature map has better quality/time ratio than other architectures.

The memory required by FCM algorithm and by Kohonen feature map for classifying six categories of tissues in one 256 by 256 image is about 1.6 Mbytes $\left(6^{*} 4 * 64 \mathrm{Kbytes}\right)$ and 0.4 Mbytes, respectively. It indicates that Kohonen feature map is less memory-consuming than FCM. And the method combined Kohonen Self Organizing Map with FCM saves more than $40 \%$ of time needed for training and adjusting the parameters for image segmentation when compared with the method of FCM classification.

In order to prove that the results of the segmentation can be applied to clinical usage, we compared the segmented tissues in test images to that in the reference PAIR images. The result shows that it can not only match the reference image, but also pass the validation of the clinician.

Kohonen feature map based image segmentation method can reduce the deviation caused by the interobserver or intraobserver and has an excellent reliability for different volunteers. These results show that the method proposed in this study can be used to assist the clinician to delineate the tissue of interest more efficiently and correctly. And the result of segmentation can further be applied for the measurement and volume rendering, etc.

\section{References}

[1] R. L. Cannon, J. B. Dave, and J. C. Bezdek, "Efficient impletememtation of the fuzzy c-means clustering algorithms", IEEE Trans. on Pattern Analysis and Machine Intelligence, Vol. PAMI-8, No. 2, pp. 248$255,1986$.

[2] T. Kohonen, Self-Organization and Associative Memory, Spinger-Verlag, New York, 1984

[3] Andrew Simmons, Gareth J. Barker, Paul S. Tofts, Achim Gass, and Simon R. Arridge, "A method for visualization of MRI partial volume regions-PAIR(PArtial volume sensitised Inversion Recovery imaging), Magnetic Resonance Imaging, Vol. 12, No. 5, pp. 821-826, 1994

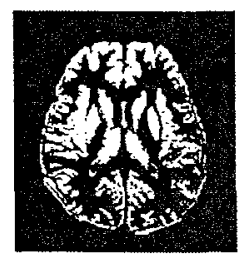

gray matter

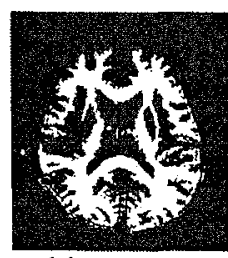

white matter

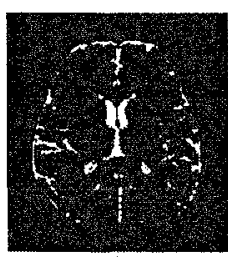

CSF
Fig. 3 The results obtained by the Kohonen feature map with 8 by 8 output layer nodes.
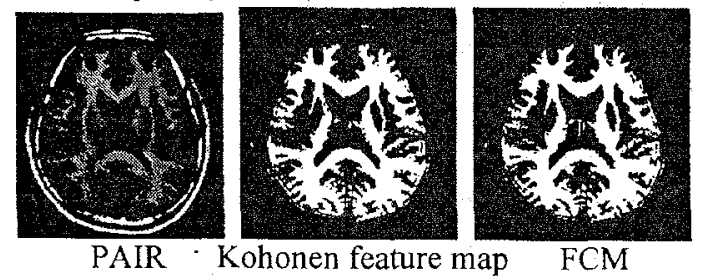

Fig. 4 The reference image and segmented images.

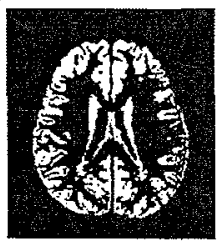

gray matter

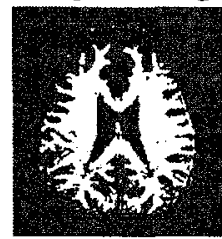

white matter

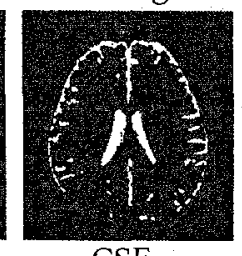

CSF
Fig. 5 The result of image segmentation of different slices of the same volunteer.

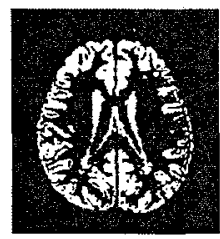

gray matter

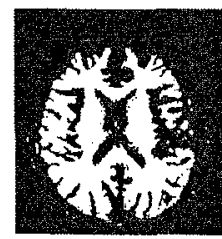

white matter

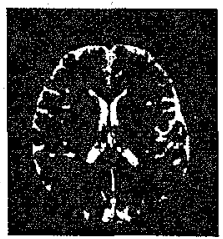

CSF
Fig. 6 The result of image segmentation of image slices of different volunteer. 